\title{
Forced oscillation of certain fractional differential equations
}

\author{
Da-Xue Chen ${ }^{1 *}$, Pei-Xin Qu ${ }^{2}$ and Yong-Hong Lan ${ }^{3}$
}

"Correspondence: cdx2003@163.com

${ }^{1}$ College of Science, Hunan Institute of Engineering, 88 East Fuxing Road, Xiangtan, Hunan, 411104, P.R. China Full list of author information is available at the end of the article

\begin{abstract}
The paper deals with the forced oscillation of the fractional differential equation

$$
\left(D_{a}^{a} x\right)(t)+f_{1}(t, x(t))=v(t)+f_{2}(t, x(t)) \text { for } t>a \geq 0
$$

with the initial conditions $\left(D_{a}^{q-k} x\right)(a)=b_{k}(k=1,2, \ldots, m-1)$ and $\lim _{t \rightarrow a^{+}}\left(I_{a}^{m-a} x\right)(t)=b_{m}$, where $D_{a}^{q} x$ is the Riemann-Liouville fractional derivative of order $q$ of $x, m-1<q \leq m$, $m \geq 1$ is an integer, $l_{a}^{m-q} x$ is the Riemann-Liouville fractional integral of order $m-q$ of $x$, and $b_{k}(k=1,2, \ldots, m)$ are/is constants/constant. We obtain some oscillation theorems for the equation by reducing the fractional differential equation to the equivalent Volterra fractional integral equation and by applying Young's inequality. We also establish some new oscillation criteria for the equation when the Riemann-Liouville fractional operator is replaced by the Caputo fractional operator. The results obtained here improve and extend some existing results. An example is given to illustrate our theoretical results.
\end{abstract}

MSC: $34 \mathrm{A0} 8 ; 34 \mathrm{C} 10$

Keywords: forced oscillation; fractional derivative; fractional differential equation

\section{Introduction}

The aim of the paper is to establish several oscillation theorems for forced fractional differential equation with initial conditions of the form

$$
\left.\begin{array}{l}
\left(D_{a}^{q} x\right)(t)+f_{1}(t, x(t))=v(t)+f_{2}(t, x(t)), \quad t>a \geq 0 \\
\left(D_{a}^{q-k} x\right)(a)=b_{k} \quad(k=1,2, \ldots, m-1), \quad \lim _{t \rightarrow a^{+}}\left(I_{a}^{m-q} x\right)(t)=b_{m}
\end{array}\right\}
$$

where $D_{a}^{q} x$ is the Riemann-Liouville fractional derivative of order $q$ of $x, m-1<q \leq m$, $m \geq 1$ is an integer, $I_{a}^{m-q} x$ is the Riemann-Liouville fractional integral of order $m-q$ of $x$, $b_{k}(k=1,2, \ldots, m)$ are/is constants/constant, $f_{i}:[a, \infty) \times \mathbb{R} \rightarrow \mathbb{R}(i=1,2)$ are continuous functions, and $v:[a, \infty) \rightarrow \mathbb{R}$ is a continuous function.

By a solution of (1.1), we mean a nontrivial function $x \in C([a, \infty), \mathbb{R})$ which has the property $D_{a}^{q} x \in C([a, \infty), \mathbb{R})$ and satisfies $(1.1)$ for $t \geq a$. Our attention is restricted to those solutions of (1.1) which exist on $[a, \infty)$ and satisfy $\sup \left\{|x(t)|: t>t_{*}\right\}>0$ for any $t_{*} \geq a$. A solution $x$ of (1.1) is said to be oscillatory if it is neither eventually positive nor eventually negative; otherwise, it is nonoscillatory. Equation (1.1) is said to be oscillatory if all its solutions are oscillatory.

(c) 2013 Chen et al.; licensee Springer. This is an Open Access article distributed under the terms of the Creative Commons Attribution License (http://creativecommons.org/licenses/by/2.0), which permits unrestricted use, distribution, and reproduction in any medium, provided the original work is properly cited. 
Differential equations of fractional order have recently proved to be valuable tools in the modeling of many phenomena in various fields of science and engineering. Indeed, we can find numerous applications in viscoelasticity, electrochemistry, control, porous media, electromagnetic, etc.; see, for example, [1-6]. There has been a significant development in ordinary and partial differential equations involving both Riemann-Liouville and Caputo fractional derivatives in recent years. The books on the subject of fractional integrals and fractional derivatives by Diethelm [7], Miller and Ross [8], Podlubny [9] and Kilbas et al. [10] summarize and organize much of fractional calculus and many of theories and applications of fractional differential equations. Many papers have studied some aspects of fractional differential equations such as the existence and uniqueness of solutions to Cauchy type problems, the methods for explicit and numerical solutions, and the stability of solutions, and we refer to [11-18] and the references quoted therein.

However, to the best of our knowledge, very little is known regarding the oscillation of fractional differential equations up to now. Recently, Chen [19] established some oscillation criteria for the fractional differential equation

$$
\left[r(t)\left(D_{-}^{q} x\right)^{\eta}(t)\right]^{\prime}-p(t) f\left(\int_{t}^{\infty}(s-t)^{-q} x(s) \mathrm{d} s\right)=0 \quad \text { for } t>0
$$

where $q \in(0,1)$ is a constant, $\eta>0$ is a quotient of odd positive integers, $D_{-}^{q} x$ is the Liouville right-sided fractional derivative of order $q$ of $x$ defined by

$$
\left(D_{-}^{q} x\right)(t):=-\frac{1}{\Gamma(1-q)} \frac{\mathrm{d}}{\mathrm{d} t} \int_{t}^{\infty}(s-t)^{-q} x(s) \mathrm{d} s \quad \text { for } t>0,
$$

here $\Gamma$ is the gamma function defined by

$$
\Gamma(t):=\int_{0}^{\infty} s^{t-1} e^{-s} \mathrm{~d} s \quad \text { for } t>0 .
$$

For details of the Liouville fractional integrals and fractional derivatives, one can refer to [10, Sections 2.2 and 2.3].

Grace et al. [20] discussed the oscillation of a forced fractional differential equation with initial conditions of the form (1.1) under the conditions

$$
x f_{i}(t, x)>0 \text { for } i=1,2, x \neq 0 \text { and } t \geq a,
$$

and

$$
\left|f_{1}(t, x)\right| \geq p_{1}(t)|x|^{\beta} \quad \text { and } \quad\left|f_{2}(t, x)\right| \leq p_{2}(t)|x|^{\gamma} \quad \text { for } x \neq 0 \text { and } t \geq a \text {, }
$$

where $p_{1}, p_{2} \in C([a, \infty),(0, \infty))$ and $\beta, \gamma>0$ are constants. Grace et al. gave several oscillation results for (1.1) by reducing the equation to the equivalent Volterra fractional integral equation (see [7, Lemma 5.2])

$$
\begin{aligned}
x(t)= & \sum_{k=1}^{m} \frac{b_{k}(t-a)^{q-k}}{\Gamma(q-k+1)} \\
& +\frac{1}{\Gamma(q)} \int_{a}^{t}(t-s)^{q-1}\left[v(s)+f_{2}(s, x(s))-f_{1}(s, x(s))\right] \mathrm{d} s \quad \text { for } t>a
\end{aligned}
$$


when $\beta>1=\gamma, \beta=1>\gamma>0$ and $\beta>1>\gamma>0$, respectively. The results are also stated when the Riemann-Liouville fractional operator is replaced by the Caputo fractional operator.

Obviously, Grace et al. [20] did not consider the cases $\beta>\gamma>1$ and $1>\beta>\gamma>0$ for (1.1). In this paper, we establish several oscillation criteria for (1.1) under the conditions (1.2), (1.3) and $\beta>\gamma>0$ by using Young's inequality. Furthermore, we obtain some oscillation theorems for (1.1) without the condition (1.3) but with the condition (1.2) and the following conditions:

$$
\left|f_{1}(t, x)\right| \leq p_{1}(t)|x|^{\beta} \quad \text { and } \quad\left|f_{2}(t, x)\right| \geq p_{2}(t)|x|^{\gamma} \quad \text { for } x \neq 0 \text { and } t \geq a \text {, }
$$

where $p_{1}, p_{2} \in C([a, \infty),(0, \infty))$ and $\beta, \gamma>0$ are constants. We also get some new oscillatory properties of (1.1) when the Riemann-Liouville fractional operator is replaced by the Caputo fractional operator. Our results improve and extend some of those in [20].

\section{Preliminaries and a lemma}

In this section, we recall several definitions of fractional integrals and fractional derivatives and the well-known Young's inequality, which will be used throughout this paper. More details on fractional calculus can be found in [7-10].

Definition 2.1 [7] The Riemann-Liouville fractional integral of order $q>0$ of a function $x:[a, \infty) \rightarrow \mathbb{R}$ is defined by

$$
\left(I_{a}^{q} x\right)(t):=\frac{1}{\Gamma(q)} \int_{a}^{t}(t-s)^{q-1} x(s) \mathrm{d} s
$$

provided the right-hand side is pointwise defined on $[a, \infty)$, where $\Gamma$ is the gamma function. Furthermore, we set $I_{a}^{0} x:=x$.

Definition 2.2 [7] The Riemann-Liouville fractional derivative of order $q>0$ of a function $x:[a, \infty) \rightarrow \mathbb{R}$ is defined by

$$
\left(D_{a}^{q} x\right)(t):=\frac{\mathrm{d}^{m}}{\mathrm{~d} t^{m}}\left(I_{a}^{m-q} x\right)(t)
$$

provided the right-hand side is pointwise defined on $[a, \infty)$, where $m-1<q \leq m$ and $m \geq 1$ is an integer. Furthermore, we set $D_{a}^{0} x:=x$.

Definition 2.3 [7] The Caputo fractional derivative of order $q>0$ of a function $x$ : $[a, \infty) \rightarrow \mathbb{R}$ is defined by

$$
\left({ }^{C} D_{a}^{q} x\right)(t):=\left(I_{a}^{m-q} x^{(m)}\right)(t)
$$

provided the right-hand side is pointwise defined on $[a, \infty)$, where $m-1<q \leq m, m \geq 1$ is an integer and $x^{(m)}$ denotes the usual derivative of integer order $m$ of $x$. Furthermore, we set ${ }^{C} D_{a}^{0} x:=x$. 
Lemma 2.1 (Young's inequality) (i) Let $X, Y \geq 0, u>1$ and $\frac{1}{u}+\frac{1}{v}=1$, then $X Y \leq \frac{1}{u} X^{u}+\frac{1}{v} Y^{v}$, where the equality holds if and only if $Y=X^{u-1}$.

(ii) Let $X \geq 0, Y>0,0<u<1$ and $\frac{1}{u}+\frac{1}{v}=1$, then $X Y \geq \frac{1}{u} X^{u}+\frac{1}{v} Y^{v}$, where the equality holds if and only if $Y=X^{u-1}$.

\section{Main results}

Theorem 3.1 Suppose that (1.2) and (1.3) hold with $\beta>\gamma$. If

$$
\liminf _{t \rightarrow \infty} t^{1-q} \int_{T}^{t}(t-s)^{q-1}[v(s)+H(s)] \mathrm{d} s=-\infty
$$

and

$$
\limsup _{t \rightarrow \infty} t^{1-q} \int_{T}^{t}(t-s)^{q-1}[v(s)-H(s)] \mathrm{d} s=\infty
$$

for every sufficiently large $T$, where $H(s):=(\beta / \gamma-1)\left[\gamma p_{2}(s) / \beta\right]^{\beta /(\beta-\gamma)} p_{1}^{\gamma /(\gamma-\beta)}(s)$, then every solution of (1.1) is oscillatory.

Proof Let $x$ be a nonoscillatory solution of (1.1). Firstly, we suppose that $x$ is an eventually positive solution of (1.1). Then there exists $T_{1}>a$ such that $x(t)>0$ for $t \geq T_{1}$. Let $s \geq T_{1}$ and take $X=|x|^{\gamma}(s), Y=\gamma p_{2}(s) /\left(\beta p_{1}(s)\right), u=\beta / \gamma$ and $v=\beta /(\beta-\gamma)$, then from Part (i) of Lemma 2.1 we conclude

$$
\begin{aligned}
p_{2}(s)|x|^{\gamma}(s)-p_{1}(s)|x|^{\beta}(s) & \\
= & \frac{\beta p_{1}(s)}{\gamma}\left[|x|^{\gamma}(s) \frac{\gamma p_{2}(s)}{\beta p_{1}(s)}-\frac{1}{\beta / \gamma}\left(|x|^{\gamma}(s)\right)^{\beta / \gamma}\right] \\
= & \frac{\beta p_{1}(s)}{\gamma}\left[X Y-\frac{1}{u} X^{u}\right] \leq \frac{\beta p_{1}(s)}{\gamma} \frac{1}{v} Y^{v}=H(s) \quad \text { for } s \geq T_{1},
\end{aligned}
$$

where $H$ is defined as in Theorem 3.1. From (1.4), (1.2), (1.3) and (3.3), we obtain

$$
\begin{aligned}
\Gamma(q) x(t)= & \Gamma(q) \sum_{k=1}^{m} \frac{b_{k}(t-a)^{q-k}}{\Gamma(q-k+1)}+\int_{a}^{T_{1}}(t-s)^{q-1}\left[v(s)+f_{2}(s, x(s))-f_{1}(s, x(s))\right] \mathrm{d} s \\
& +\int_{T_{1}}^{t}(t-s)^{q-1}\left[v(s)+f_{2}(s, x(s))-f_{1}(s, x(s))\right] \mathrm{d} s \\
\leq & \Phi(t)+\Psi\left(t, T_{1}\right)+\int_{T_{1}}^{t}(t-s)^{q-1}\left[v(s)+p_{2}(s) x^{\gamma}(s)-p_{1}(s) x^{\beta}(s)\right] \mathrm{d} s \\
\leq & \Phi(t)+\Psi\left(t, T_{1}\right)+\int_{T_{1}}^{t}(t-s)^{q-1}[v(s)+H(s)] \mathrm{d} s \quad \text { for } t \geq T_{1},
\end{aligned}
$$

where

$$
\Phi(t):=\Gamma(q) \sum_{k=1}^{m} \frac{b_{k}(t-a)^{q-k}}{\Gamma(q-k+1)}
$$


and

$$
\Psi\left(t, T_{1}\right):=\int_{a}^{T_{1}}(t-s)^{q-1}\left[v(s)+f_{2}(s, x(s))-f_{1}(s, x(s))\right] \mathrm{d} s .
$$

Multiplying (3.4) by $t^{1-q}$, we have, for $t \geq T_{1}$,

$$
0<t^{1-q} \Gamma(q) x(t) \leq t^{1-q} \Phi(t)+t^{1-q} \Psi\left(t, T_{1}\right)+t^{1-q} \int_{T_{1}}^{t}(t-s)^{q-1}[v(s)+H(s)] \mathrm{d} s .
$$

Take $T_{2}>T_{1}$. Next, we consider the cases $0<q \leq 1$ and $q>1$, respectively.

Case (i). Let $0<q \leq 1$. Then we get $m=1, \Phi(t)=b_{1}(t-a)^{q-1}$,

$$
\left|t^{1-q} \Phi(t)\right|=\left|b_{1}\right| t^{1-q}(t-a)^{q-1} \leq\left|b_{1}\right|\left(\frac{T_{2}}{T_{2}-a}\right)^{1-q}:=c_{1}\left(T_{2}\right) \quad \text { for } t \geq T_{2}
$$

and

$$
\begin{aligned}
\left|t^{1-q} \Psi\left(t, T_{1}\right)\right| & =\left|t^{1-q} \int_{a}^{T_{1}}(t-s)^{q-1}\left[v(s)+f_{2}(s, x(s))-f_{1}(s, x(s))\right] \mathrm{d} s\right| \\
& \leq \int_{a}^{T_{1}} t^{1-q}(t-s)^{q-1}\left|v(s)+f_{2}(s, x(s))-f_{1}(s, x(s))\right| \mathrm{d} s \\
& \leq \int_{a}^{T_{1}}\left(\frac{T_{2}}{T_{2}-s}\right)^{1-q}\left|v(s)+f_{2}(s, x(s))-f_{1}(s, x(s))\right| \mathrm{d} s \\
& :=c_{2}\left(T_{1}, T_{2}\right) \quad \text { for } t \geq T_{2} .
\end{aligned}
$$

It follows from (3.7)-(3.9) that $t^{1-q} \int_{T_{1}}^{t}(t-s)^{q-1}[v(s)+H(s)] \mathrm{d} s>-\left[c_{1}\left(T_{2}\right)+c_{2}\left(T_{1}, T_{2}\right)\right]$ for $t \geq$ $T_{2}$. Therefore, we find $\liminf _{t \rightarrow \infty} t^{1-q} \int_{T_{1}}^{t}(t-s)^{q-1}[v(s)+H(s)] \mathrm{d} s \geq-\left[c_{1}\left(T_{2}\right)+c_{2}\left(T_{1}, T_{2}\right)\right]>$ $-\infty$, which contradicts (3.1).

Case (ii). Let $q>1$. Then we have $m \geq 2$,

$$
\begin{aligned}
\left|t^{1-q} \Phi(t)\right| & =\left|t^{1-q} \Gamma(q) \sum_{k=1}^{m} \frac{b_{k}(t-a)^{q-k}}{\Gamma(q-k+1)}\right| \\
& \leq \Gamma(q) \sum_{k=1}^{m} \frac{\left|b_{k}\right| t^{1-q}(t-a)^{q-k}}{\Gamma(q-k+1)} \leq \Gamma(q) \sum_{k=1}^{m} \frac{\left|b_{k}\right|\left(T_{2}-a\right)^{1-k}}{\Gamma(q-k+1)} \\
& :=c_{3}\left(T_{2}\right) \quad \text { for } t \geq T_{2}
\end{aligned}
$$

and

$$
\begin{aligned}
\left|t^{1-q} \Psi\left(t, T_{1}\right)\right| & =\left|t^{1-q} \int_{a}^{T_{1}}(t-s)^{q-1}\left[v(s)+f_{2}(s, x(s))-f_{1}(s, x(s))\right] \mathrm{d} s\right| \\
& \leq \int_{a}^{T_{1}} t^{1-q}(t-s)^{q-1}\left|v(s)+f_{2}(s, x(s))-f_{1}(s, x(s))\right| \mathrm{d} s \\
& \leq \int_{a}^{T_{1}}\left|v(s)+f_{2}(s, x(s))-f_{1}(s, x(s))\right| \mathrm{d} s:=c_{4}\left(T_{1}\right) \quad \text { for } t \geq T_{2} .
\end{aligned}
$$


From (3.7), (3.10) and (3.11), we conclude $t^{1-q} \int_{T_{1}}^{t}(t-s)^{q-1}[v(s)+H(s)] \mathrm{d} s>-\left[c_{3}\left(T_{2}\right)+\right.$ $\left.c_{4}\left(T_{1}\right)\right]$ for $t \geq T_{2}$. Hence, we obtain $\liminf _{t \rightarrow \infty} t^{1-q} \int_{T_{1}}^{t}(t-s)^{q-1}[v(s)+H(s)] \mathrm{d} s \geq-\left[c_{3}\left(T_{2}\right)+\right.$ $\left.c_{4}\left(T_{1}\right)\right]>-\infty$, which contradicts (3.1).

Finally, we assume that $x$ is an eventually negative solution of (1.1). Then a similar argument leads to a contradiction with (3.2). The proof is complete.

Remark 3.1 In [20], the plus sign ' + ' in (2.9) in Theorem 2.2, (2.13) in Theorem 2.3, (2.17) in Theorem 2.4, (3.6) in Theorem 3.2, (3.8) in Theorem 3.3 and (3.10) in Theorem 3.4 should be the minus sign '-'.

Remark 3.2 Theorems 2.2 and 2.3 in [20] are the special cases of our Theorem 3.1 with $\beta>1=\gamma$ and $\beta=1>\gamma>0$, respectively. Our Theorem 3.1 improves and extends the results of Theorems 2.2-2.4 in [20] since these theorems did not include the cases $\beta>\gamma>1$ and $1>\beta>\gamma>0$ for (1.1).

The following example shows that the condition (3.1) cannot be dropped.

Example 3.1 Consider the Riemann-Liouville fractional differential equation

$$
\left.\begin{array}{rl}
\left(D_{0}^{q} x\right)(t)+x^{5}(t) \ln (\mathrm{e}+t)= & \frac{2 t^{2-q}}{\Gamma(3-q)}+\left(t^{10}-t^{2 / 3}\right) \ln (\mathrm{e}+t) \\
& +x^{1 / 3}(t) \ln (\mathrm{e}+t), \quad t>0, \\
\lim _{t \rightarrow 0^{+}}\left(I_{0}^{1-q} x\right)(t)=0, &
\end{array}\right\}
$$

where $0<q<1$.

In (3.12), $a=0, m=1, f_{1}(t, x)=x^{5} \ln (\mathrm{e}+t), v(t)=\frac{2 t^{2-q}}{\Gamma(3-q)}+\left(t^{10}-t^{2 / 3}\right) \ln (\mathrm{e}+t), f_{2}(t, x)=$ $x^{1 / 3} \ln (\mathrm{e}+t)$ and $b_{1}=0$. Taking $p_{1}(t)=p_{2}(t)=\ln (\mathrm{e}+t), \beta=5$ and $\gamma=1 / 3$, we find that the conditions (1.2) and (1.3) are satisfied. But the condition (3.1) is not satisfied since for every sufficiently large $T \geq 1$ and all $t \geq T$, we have $v(t)>0$ and

$$
\begin{aligned}
\liminf _{t \rightarrow \infty} t^{1-q} \int_{T}^{t}(t-s)^{q-1}[v(s)+H(s)] \mathrm{d} s & \geq \liminf _{t \rightarrow \infty} t^{1-q} \int_{T}^{t}(t-s)^{q-1} H(s) \mathrm{d} s \\
& =\liminf _{t \rightarrow \infty} t^{1-q} \int_{T}^{t}(t-s)^{q-1} 15^{-15 / 14} 14 \ln (\mathrm{e}+s) \mathrm{d} s \\
& \geq \liminf _{t \rightarrow \infty} t^{1-q} \int_{T}^{t}(t-s)^{q-1} 15^{-15 / 14} 14 \mathrm{~d} s \\
& =\liminf _{t \rightarrow \infty} \frac{15^{-15 / 14} 14 t^{1-q}(t-T)^{q}}{q} \\
& =\infty,
\end{aligned}
$$

where $H$ is defined as in Theorem 3.1. Taking $x(t)=t^{2}$, by Definition 2.1 we get

$$
\left(I_{0}^{1-q} x\right)(t)=\frac{1}{\Gamma(1-q)} \int_{0}^{t}(t-s)^{-q} s^{2} \mathrm{~d} s
$$


Integrating by parts twice, we obtain

$$
\left(I_{0}^{1-q} x\right)(t)=\frac{1}{\Gamma(1-q)} \frac{2 t^{3-q}}{(1-q)(2-q)(3-q)} .
$$

Therefore, by Definition 2.2 we conclude

$$
\left(D_{0}^{q} x\right)(t)=\frac{\mathrm{d}}{\mathrm{d} t}\left(I_{0}^{1-q} x\right)(t)=\frac{1}{\Gamma(1-q)} \frac{2 t^{2-q}}{(1-q)(2-q)}=\frac{2 t^{2-q}}{\Gamma(3-q)},
$$

which implies that $x(t)=t^{2}$ satisfies the first equality in (3.12). From (3.13) we get $\lim _{t \rightarrow 0^{+}}\left(I_{0}^{1-q} x\right)(t)=0$, which yields that $x(t)=t^{2}$ satisfies the second equality in (3.12). Hence, $x(t)=t^{2}$ is a nonoscillatory solution of (3.12).

Next, we consider the case when (1.5) holds, which was not considered in [20].

Theorem 3.2 Let $q \geq 1$ and suppose that (1.2) and (1.5) hold with $\beta<\gamma$. If

$$
\limsup _{t \rightarrow \infty} t^{1-q} \int_{T}^{t}(t-s)^{q-1}[v(s)+H(s)] \mathrm{d} s=\infty
$$

and

$$
\liminf _{t \rightarrow \infty} t^{1-q} \int_{T}^{t}(t-s)^{q-1}[v(s)-H(s)] \mathrm{d} s=-\infty
$$

for every sufficiently large $T$, where $H$ is defined as in Theorem 3.1, then every bounded solution of (1.1) is oscillatory.

Proof Let $x$ be a bounded nonoscillatory solution of (1.1). Then there exist constants $M_{1}$ and $M_{2}$ such that

$$
M_{1} \leq x(t) \leq M_{2} \quad \text { for } t \geq a
$$

Firstly, we suppose that $x$ is a bounded eventually positive solution of (1.1). Then there exists $T_{1}>a$ such that $x(t)>0$ for $t \geq T_{1}$. Similar to the proof of (3.3), by Part (ii) of Lemma 2.1 we find

$$
p_{2}(s)|x|^{\gamma}(s)-p_{1}(s)|x|^{\beta}(s) \geq H(s) \quad \text { for } s \geq T_{1},
$$

where $H$ is defined as in Theorem 3.1. Define $\Phi$ and $\Psi$ as in (3.5) and (3.6), respectively. Similar to the proof of (3.7), from (1.4), (1.2), (1.5) and (3.17), we get, for $t \geq T_{1}$,

$$
t^{1-q} \Gamma(q) x(t) \geq t^{1-q} \Phi(t)+t^{1-q} \Psi\left(t, T_{1}\right)+t^{1-q} \int_{T_{1}}^{t}(t-s)^{q-1}[v(s)+H(s)] \mathrm{d} s .
$$

Take $T_{2}>T_{1}$. Next, we consider the cases $q=1$ and $q>1$, respectively.

Case (i). Let $q=1$. Then (3.8) and (3.9) are still true. From (3.16), (3.8), (3.9) and (3.18), we conclude $M_{2} \Gamma(q) \geq-c_{1}\left(T_{2}\right)-c_{2}\left(T_{1}, T_{2}\right)+t^{1-q} \int_{T_{1}}^{t}(t-s)^{q-1}[v(s)+H(s)] \mathrm{d} s$ for $t \geq T_{2}$. Thus, 
we see $\lim \sup _{t \rightarrow \infty} t^{1-q} \int_{T_{1}}^{t}(t-s)^{q-1}[v(s)+H(s)] \mathrm{d} s \leq c_{1}\left(T_{2}\right)+c_{2}\left(T_{1}, T_{2}\right)+M_{2} \Gamma(q)<\infty$, which contradicts (3.14).

Case (ii). Let $q>1$. Then (3.10) and (3.11) are still valid. From (3.16), (3.10), (3.11) and (3.18), we conclude $M_{2} \Gamma(q) t^{1-q} \geq-c_{3}\left(T_{2}\right)-c_{4}\left(T_{1}\right)+t^{1-q} \int_{T_{1}}^{t}(t-s)^{q-1}[v(s)+H(s)] \mathrm{d} s$ for $t \geq$ $T_{2}$. Since $\lim _{t \rightarrow \infty} t^{1-q}=0$, we obtain $\limsup _{t \rightarrow \infty} t^{1-q} \int_{T_{1}}^{t}(t-s)^{q-1}[v(s)+H(s)] \mathrm{d} s \leq c_{3}\left(T_{2}\right)+$ $c_{4}\left(T_{1}\right)<\infty$, which contradicts (3.14).

Finally, we suppose that $x$ is a bounded eventually negative solution of (1.1). Then a similar argument leads to a contradiction with (3.15). The proof is complete.

\section{Results with the Caputo fractional derivative}

The Riemann-Liouville fractional derivatives played an important role in the development of the theory of fractional derivatives and integrals and for their applications in pure mathematics. But it turns out that the Riemann-Liouville derivatives have certain disadvantages when trying to model real-world phenomena with fractional differential equations. When comparing the Riemann-Liouville definition and the Caputo definition of fractional derivatives, we will see this second one seems to be better suited to such tasks. The main advantages of the Caputo fractional derivatives is that the initial conditions for fractional differential equations with Caputo fractional derivatives take on the same form as for integer-order differential equations, i.e., they contain the limit values of integer-order derivatives of unknown functions at the lower terminal $t=a$.

In this section, we study the oscillation of (1.1) when the Riemann-Liouville fractional operator is replaced by the Caputo fractional operator, i.e., the oscillation of the initial value problem

$$
\left.\begin{array}{l}
\left({ }^{C} D_{a}^{q} x\right)(t)+f_{1}(t, x(t))=v(t)+f_{2}(t, x(t)), \quad t>a \geq 0, \\
x^{(k)}(a)=b_{k} \quad(k=0,1, \ldots, m-1),
\end{array}\right\}
$$

where ${ }^{C} D_{a}^{q} x$ is the Caputo fractional derivative of order $q$ of $x$ defined by (2.3), $m-1<q \leq$ $m, m \geq 1$ is an integer, $b_{k}(k=0,1, \ldots, m-1)$ are/is constants/constant, $f_{i}:[a, \infty) \times \mathbb{R} \rightarrow$ $\mathbb{R}(i=1,2)$ are continuous functions, and $v:[a, \infty) \rightarrow \mathbb{R}$ is a continuous function. The corresponding Volterra fractional integral equation (see [7, Lemma 6.2]) becomes

$$
x(t)=\sum_{k=0}^{m-1} \frac{b_{k}(t-a)^{k}}{k !}+\frac{1}{\Gamma(q)} \int_{a}^{t}(t-s)^{q-1}\left[v(s)+f_{2}(s, x(s))-f_{1}(s, x(s))\right] \mathrm{d} s \quad \text { for } t>a .
$$

Similar to the proof of Theorems 3.1 and 3.2, we can prove the following theorems.

Theorem 4.1 Suppose that (1.2) and (1.3) hold with $\beta>\gamma$. If

$$
\liminf _{t \rightarrow \infty} t^{1-m} \int_{T}^{t}(t-s)^{q-1}[v(s)+H(s)] \mathrm{d} s=-\infty
$$

and

$$
\limsup _{t \rightarrow \infty} t^{1-m} \int_{T}^{t}(t-s)^{q-1}[v(s)-H(s)] \mathrm{d} s=\infty
$$

for every sufficiently large $T$, where $H$ is defined as in Theorem 3.1, then every solution of (4.1) is oscillatory. 
Theorem 4.2 Let $q \geq 1$ and suppose that (1.2) and (1.5) hold with $\beta<\gamma$. If

$$
\limsup _{t \rightarrow \infty} t^{1-m} \int_{T}^{t}(t-s)^{q-1}[v(s)+H(s)] \mathrm{d} s=\infty
$$

and

$$
\liminf _{t \rightarrow \infty} t^{1-m} \int_{T}^{t}(t-s)^{q-1}[v(s)-H(s)] \mathrm{d} s=-\infty
$$

for every sufficiently large $T$, where $H$ is defined as in Theorem 3.1, then every bounded solution of (4.1) is oscillatory.

Remark 4.1 Theorems 3.2 and 3.3 in [20] are the special cases of our Theorem 4.1 with $\beta>1=\gamma$ and $\beta=1>\gamma>0$, respectively. Our Theorem 4.1 improves and extends the results of Theorems 3.2-3.4 in [20] since these theorems did not include the cases $\beta>\gamma>1$ and $1>\beta>\gamma>0$ for (4.1). The case considered in our Theorem 4.2 was not discussed in [20] and hence our Theorem 4.2 is a new result.

\section{Competing interests}

The authors declare that they have no competing interests.

\section{Authors' contributions}

The first author discovered the topic and the main ideas for the proof of the paper and made the actual writing. All authors discussed the paper together. The second and the third authors discovered some helpful ideas for the proof of this paper and checked the proof of the paper. All authors read and approved the final manuscript.

\section{Author details}

${ }^{1}$ College of Science, Hunan Institute of Engineering, 88 East Fuxing Road, Xiangtan, Hunan, 411104, P.R. China. ${ }^{2}$ School of Information \& Engineering, Henan Institute of Science and Technology, Xinxiang, Henan 453003, P.R. China. ${ }^{3}$ School of Information Engineering, Xiangtan University, Xiangtan, Hunan, 411105, P.R. China.

\section{Acknowledgements}

Dedicated to Professor Hari M Srivastava.

The authors are very grateful to the anonymous referees for their valuable suggestions and comments, which helped the authors to improve the previous manuscript of the article. This work was supported by the National Natural Science Foundation of P.R. China (Grants No. 11271311 and No. 61104072) and the Natural Science Foundation of Hunan of P.R. China (Grant No. 11JJ3010).

Received: 2 December 2012 Accepted: 19 April 2013 Published: 2 May 2013

\section{References}

1. Das, S: Functional Fractional Calculus for System Identification and Controls. Springer, New York (2008)

2. Diethelm, K, Freed, AD: On the solution of nonlinear fractional order differential equations used in the modeling of viscoplasticity. In: Keil, F, Mackens, W, Vob, H, Werther, J (eds.) Scientific Computing in Chemical Engineering II: Computational Fluid Dynamics, Reaction Engineering and Molecular Properties, pp. 217-224. Springer, Heidelberg (1999)

3. Gaul, L, Klein, P, Kempfle, S: Damping description involving fractional operators. Mech. Syst. Signal Process. 5, 81-88 (1991). doi:10.1016/0888-3270(91)90016-X

4. Glöckle, WG, Nonnenmacher, TF: A fractional calculus approach to self-similar protein dynamics. Biophys. J. 68, 46-53 (1995). doi:10.1016/S0006-3495(95)80157-8

5. Mainardi, F: Fractional calculus: some basic problems in continuum and statistical mechanics. In: Carpinteri, A, Mainardi, F (eds.) Fractals and Fractional Calculus in Continuum Mechanics, pp. 291-348. Springer, Vienna (1997)

6. Metzler, R, Schick, W, Kilian, HG, Nonnenmacher, TF: Relaxation in filled polymers: a fractional calculus approach. J. Chem. Phys. 103, 7180-7186 (1995). doi:10.1063/1.470346

7. Diethelm, K: The Analysis of Fractional Differential Equations. Springer, Berlin (2010)

8. Miller, KS, Ross, B: An Introduction to the Fractional Calculus and Fractional Differential Equations. Wiley, New York (1993)

9. Podlubny, I: Fractional Differential Equations. Academic Press, San Diego (1999)

10. Kilbas, AA, Srivastava, HM, Trujillo, J: Theory and Applications of Fractional Differential Equations. Elsevier, Amsterdam (2006) 
11. Muslim, M: Existence and approximation of solutions to fractional differential equations. Math. Comput. Model. 49, 1164-1172 (2009). doi:10.1016/j.mcm.2008.07.013

12. Saadatmandi, A, Dehghan, M: A new operational matrix for solving fractional-order differential equations. Comput. Math. Appl. 59, 1326-1336 (2010). doi:10.1016/j.camwa.2009.07.006

13. Zhou, Y, Jiao, F, Li, J: Existence and uniqueness for $p$-type fractional neutral differential equations. Nonlinear Anal. 71, 2724-2733 (2009). doi:10.1016/.na.2009.01.105

14. Ghoreishi, F, Yazdani, S: An extension of the spectral Tau method for numerical solution of multi-order fractional differential equations with convergence analysis. Comput. Math. Appl. 61, 30-43 (2011). doi:10.1016/j.camwa.2010.10.027

15. Edwards, JT, Ford, NJ, Simpson, AC: The numerical solution of linear multi-term fractional differential equations: systems of equations. J. Comput. Appl. Math. 148, 401-418 (2002). doi:10.1016/S0377-0427(02)00558-7

16. Galeone, L, Garrappa, R: Explicit methods for fractional differential equations and their stability properties. J. Comput. Appl. Math. 228, 548-560 (2009). doi:10.1016/j.cam.2008.03.025

17. Trigeassou, JC, Maamri, N, Sabatier, J, Oustaloup, A: A Lyapunov approach to the stability of fractional differential equations. Signal Process. 91, 437-445 (2011). doi:10.1016/j.sigpro.2010.04.024

18. Deng, W: Smoothness and stability of the solutions for nonlinear fractional differential equations. Nonlinear Anal. 72, 1768-1777 (2010). doi:10.1016/..na.2009.09.018

19. Chen, DX: Oscillation criteria of fractional differential equations. Adv. Differ. Equ. 2012, 1-10 (2012). doi:10.1186/1687-1847-2012-33

20. Grace, SR, Agarwal, RP, Wong, PJY, Zafer, A: On the oscillation of fractional differential equations. Fract. Calc. Appl. Anal. 15, 222-231 (2012). doi:10.2478/s13540-012-0016-1

doi:10.1186/1687-1847-2013-125

Cite this article as: Chen et al.: Forced oscillation of certain fractional differential equations. Advances in Difference Equations 2013 2013:125.

\section{Submit your manuscript to a SpringerOpen ${ }^{\circ}$ journal and benefit from:}

- Convenient online submission

- Rigorous peer review

- Immediate publication on acceptance

- Open access: articles freely available online

- High visibility within the field

- Retaining the copyright to your article 